\title{
Small intestine without mucosa as a growing vascular conduit: A porcine experimental study
}

\author{
Om P. Tucker, $\mathrm{MD}^{\mathrm{a}}$ \\ Thomas Syburra ${ }^{a}$ \\ Monique Augstburger, $\mathrm{RN}^{\mathrm{a}}$ \\ Guy van Melle, $\mathrm{PhD}^{\mathrm{b}}$ \\ Sandra Gebhard, $\mathrm{MD}^{\mathrm{c}}$ \\ Fred Bosman, MD ${ }^{\mathrm{b}}$ \\ Ludwig K. von Segesser, MD
}

From the Departments of Cardiovascular Surgery, ${ }^{\mathrm{a}}$ Statistics, ${ }^{\mathrm{b}}$ and Pathology, ${ }^{\mathrm{c}}$ University Hospital Vaudois, Centre Hospitalier Universitaire Vaudois, Lausanne, Switzerland.

Read at the Twenty-seventh Annual Meeting of The Western Thoracic Surgical Association, San Diego, Calif, June 20-23, 2001.

Received for publication June 28, 2001; revisions requested Nov 19, 2001; revisions received Jan 24, 2002; accepted for publication March 27, 2002.

Address for reprints: Om P. Tucker, MD, Department of Cardiovascular Surgery, Centre Hospitalier Universitaire Vaudois, Lausanne, Switzerland (E-mail: omptucker @ hotmail.com).

J Thorac Cardiovasc Surg 2002;124: 1165-75

Copyright () 2002 by The American Association for Thoracic Surgery

0022-5223/2002 $\$ 35.00+0 \quad \mathbf{1 2 / 6 / 1 2 5 8 1 8}$

doi: $10.1067 / \mathrm{mtc} .2002 .125818$
Objective: This study was conducted to check the feasibility of using small intestine without mucosa as a growing vascular conduit.

Method: Autologous proximal jejunum without mucosa after treatment with heparin bonding was used as a free inferior vena cava interpositional graft between the renal veins and bifurcation of inferior vena cava in 8 piglets. Intravenous ultrasonography was performed at 1 to 3 months after the operation and at autopsy.

Results: One intraoperative death was related to anesthesia. At a mean follow-up of 80 days for the 7 surviving pigs, the weight had increased by $201 \%$, from a mean of $32 \mathrm{~kg}$ to a mean of $94 \mathrm{~kg}$. The grafts had increased in length by $128 \%$, from a mean of $2.3 \mathrm{~cm}$ at implantation to a mean of $5.1 \mathrm{~cm}(P<.018)$ at explantation. In 6 animals the diameter of the graft was equal to that of the adjacent inferior vena cava. At postmortem examination, 6 grafts were patent. The single blocked graft had been patent 1 month after surgery. One graft had extensive septae inside, 2 had minor septae, 2 had microscopic septae, and 2 had no septae at all. Normal appearing adventitia, fibrous tissue, and endothelium (factor VIII-related antigen positive) lined all the grafts. In all 7 grafts, scattered proliferating fibroblasts (MIB1 positive) were observed.

Conclusions: Small intestine without mucosa remodels and acts like a live, growing, layered, endothelialized, nonthrombogenic (after re-endothelialization) vascular conduit in a growing pig. This graft material could have potential as a growing vascular conduit in children.

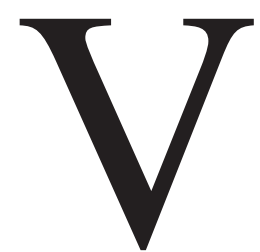

ascular conduits are used to replace malfunctioning vessel segment in both adults and children. Most of these conduits are homografts, other biologic conduits, and prosthetic grafts. Children rapidly outgrow such grafts, and repeated replacements of such conduits are necessary because of graft failure. Morbidity and mortality are considerably increased in this group of patients.

There is therefore a great demand for a live, growing, nonthrombogenic, endothelialized vascular conduit that would resist infection and degradation, but such a conduit is not available at present.

The small intestine was used as a vascular conduit more than 35 years ago and even later on, but the outcome was variable in different experimental settings. ${ }^{1,2}$ Lantz and colleagues ${ }^{3-5}$ used submucosa and the deeper layers of mucosa as an autograft, allograft, or xenograft while replacing different vessels in different experimental designs, and they demonstrated biocompatibility and high patency rates. Robotin-Johnson and associates ${ }^{6}$ reported their experience with small intestine 


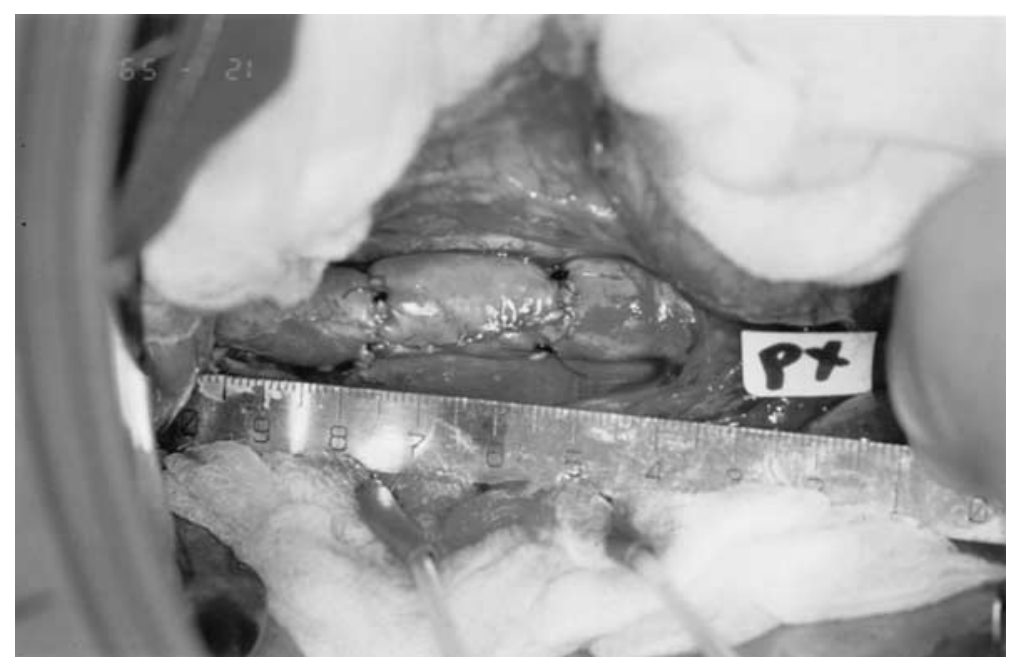

Figure 1. Plicated SIWM graft at implantation.

submucosa as a template for a growing vascular conduit while replacing superior vena cava with such a graft in piglets.

We intended to process and use small intestine without mucosa (SIWM) in such a way that after its implantation into the vascular system it would adapt itself to its new environment and work like a live, growing, endothelialized vascular conduit. We wanted to ascertain three important aspects of the graft. First, the graft had to be vital, growing along with the animal. Second, the graft had to be lined with normally functioning endothelial cells. Third, the neovascular wall had to be comparable to the native inferior vena cava (IVC) wall and well adapted to its new environment.

\section{Material and Methods}

Eight baby pigs weighing $32 \pm 4 \mathrm{~kg}$ were used in this study. All animals received humane care in compliance with the Guide for the Care and Use of Laboratory Animals prepared by the Institute of Laboratory Animal Resources, National Research Council, and published by the National Academy Press, revised 1996. The experimental protocol was approved by the Governmental Veterinary Office, Lausanne, Switzerland.

\section{Surgical Procedure and Graft Preparation}

The animals received a clear liquid diet for 1 day before the surgery and fasted overnight. Anesthesia was induced with ketamine $(10 \mathrm{mg} / \mathrm{kg})$, atropine $(0.02 \mathrm{mg} / \mathrm{kg})$, and a halothane and oxygen mixture and was maintained with halothane and oxygen.

Harvest of small intestine. Through a midline abdominal incision, a $10-\mathrm{cm}$ long piece of proximal jejunum without pedicle was harvested. The continuity of the bowel was restored in two layers with 4-0 absorbable and 4-0 nonabsorbable sutures.

Preparation of the SIWM graft. The graft was rinsed thoroughly with isotonic saline solution in the beginning. before and after processing with different solutions. The free graft was everted, and the mucosa was gently abraded with a cotton swab on back of a knife handle. The graft was then again inverted, and its lumen was exposed to $1 \%$ protamine sulfate, $0.5 \%$ glutaraldehyde, and $1 \%$ heparin solutions for 5 minutes each in that order. This is our modification of the technique originally described by Miyata and coworkers ${ }^{7}$ for rendering the graft antithrombogenic. In this method the heparin becomes bound ionically to protamine, which in turn combines covalently with the collagen by the action of glutaraldehyde. ${ }^{7}$ Heparin is then slowly released locally while endothelialization is taking place. $^{7}$

Replacement of inferior vena cava. The infrarenal IVC was mobilized, $100 \mathrm{IU} / \mathrm{kg}$ of heparin was given intravenously, vascular clamps were applied, and the middle portion of infrarenal IVC between renal veins and IVC bifurcation was replaced with $2 \mathrm{~cm}$ of the SIWM graft. The graft was plicated in 7 animals and trimmed in 1 animal to match the size of the IVC before implantation (Figure 1). In 6 animals 6-0 Prolene sutures (Ethicon, Inc, Somerville, NJ) were used for the anastomosis, whereas in the last 2 animals absorbable 6.0 monofilament sutures were used. After release of the vascular clamps, the length of the graft and its diameter at the middle of the graft were measured, the graft and the IVC were reperitonealized, and, after hemostasis was ensured, the abdomen was closed in layers without drainage. Perioperative antibiotics and low-molecular weight heparin (15,000 IU subcutaneously once a day) were given for 5 days.

\section{Diagnostic and Pathologic Examination}

Intravascular ultrasonography (IVUS) was performed in 5 animals 1 to 3 months after implantation and in all animals when they were 
TABLE 1. Weight, length, follow-up, and other variables at implantation and explantation

\begin{tabular}{llcrr}
\hline Variable & N & Mean \pm SD & Minimum & Maximum \\
\hline Follow-up (d) & 7 & $80.6 \pm 39.3$ & 15.0 & 148.0 \\
Weight at implantation (kg) & 7 & $32.3 \pm 4.4$ & 26.0 & 37.0 \\
Weight at explantation (kg) & 7 & $94.3 \pm 27.1$ & 50.0 & 13.0 \\
Increase in weight (kg) & 7 & $62.0 \pm 29.2$ & 35.1 & 128.0 \\
Increase in weight (\%) & 7 & $201.2 \pm 107.4$ & 2.0 & 3.5 \\
Length at implantation (cm) & 7 & $2.286 \pm 0.3$ & 1.0 & 2.5 \\
Length at explantation (cm) & 7 & $5.143 \pm 0.9$ & 6.0 \\
Increase in length (cm) & 7 & $2.857 \pm 0.9$ & 3.5 \\
Increase in length (\%) & 7 & $127.9 \pm 44.7$ & 0.0 & 17.0 \\
Diameter equal to IVC & 7 & $0.9 \pm 0.4$ & 0 & 1.0 \\
\hline
\end{tabular}

killed. IVUS was performed with the animals under general anesthesia, and the animals were given $100 \mathrm{IU} / \mathrm{kg}$ of heparin intravenously just before the procedure.

After IVUS studies and before explantation, the abdomen was opened through the midline incision and the infrarenal IVC and the grafts were dissected free. The length of the graft and its diameter at the center and at the site of anastomoses of the IVC and the graft were measured. The animals were then killed with sodium pentothal $(120 \mathrm{mg} / \mathrm{kg})$ and potassium chloride $(40 \mathrm{mEq})$ administered intravenously. The grafts and IVC were laid open, photographs were taken, and the specimens were fixed in $10 \%$ formalin. After fixation of the specimens, samples were taken from four quadrants from the middle of the graft, proximal, and distal anastomotic junctions for histopathologic and immunohistochemical examination with hematoxylin and eosin, van Gieson-elastin stain, factor VIII (DAKOPATTS anti-human von Willbrand factor; DAKO A/S, Glostrup, Denmark) and MIB1 (monoclonal antibody Ki-67 antigen; Immunotech Ltd, Marseille, France) monoclonal antibodies. Factor VIII and MIB1 monoclonal antibodies were used to check the endothelial differentiation and the mural proliferative fibroblasts, respectively, in the wall of the neograft.

Each animal acted as its own control as we took the measurements at the time of implantation and explantation.

\section{Data Analysis}

Weight and length at implantation and explantation were compared with the nonparametric signed-rank test for paired data. The analyses were performed with the Stata statistical software package (Stata Corporation, College Station, Tex).

\section{Results}

One pig died during the operation of anesthesia complications. The 7 surviving pigs (Table 1) were followed up for a mean period of $80 \pm 39$ days. Their weight increased by $201 \%$, from a mean of $32 \pm 4 \mathrm{~kg}$ at implantation to a mean of $94 \pm 27 \mathrm{~kg}$ at explantation. The grafts increased in length by $128 \%$, a mean increase of $2.857 \pm 0.9 \mathrm{~cm}(P<.018)$ from a mean length of $2.3 \pm 0.3 \mathrm{~cm}$ at implantation to a mean length of $5.1 \pm 0.9 \mathrm{~cm}$ at explantation. In 6 animals the diameter of the graft was equal to that of the adjacent IVC. The diameter of the single blocked graft was smaller than that of the adjoining IVC.
In 5 animals that had IVUS performed, the grafts were patent at 1 to 3 months after implantation. At explantation, IVUS studies showed that 6 grafts were patent but 1 was blocked. This occluded graft had been patent during a previous IVUS examination at 1 month after implantation but had septae in the lumen. Of the 6 patent grafts, 2 had macroscopic minor septae inside the graft, 2 had microscopic septae, and 2 had no septae at all (Figure 2).

\section{Pathologic Examination}

After preparation and before implantation, the SIWM grafts appeared macroscopically as thin-walled, pinkish, translucent tubes when filled with isotonic saline solution. Microscopically (Figures 3 and 4), the graft consisted of (from inside out) stratum compactum, muscularis mucosae, and lamina muscularis externa consisting of inner circular and outer longitudinal smooth muscle layers, subserosa, and a continuous layer of mesothelium that formed the peritoneal serosal surface.

Control IVC tissue showed the characteristics of normal large veins, including an intact endothelium supported by organized lamina, fibromuscular tissue, and elastic fibers. The tunica media of the IVC is made up of organized arrays of smooth muscle cells. The vasa vasorum are usually inconspicuous.

At autopsy, few adhesions were found between the loops of bowel. The grafts and the IVC were covered with peritoneum and could be easily dissected out from the surrounding structures in 6 animals. In 1 animal which was killed 15 days after implantation of the graft, a small organizing hematoma was found between the graft and the peritoneum. The graft was patent in this animal. The grafts were adherent to the aorta but could be dissected out. The wall of the explanted grafts (Figure 5) was slightly thinner than that of the IVC in the middle and slightly thicker near the anastomoses and the plicated area. The luminal surface in all the grafts appeared smooth and shiny (Figure 6), although some of the grafts contained septae. The suture lines were smooth and flat where absorbable sutures were used and appeared as slightly raised ridges in grafts where nonabsorbable sutures 

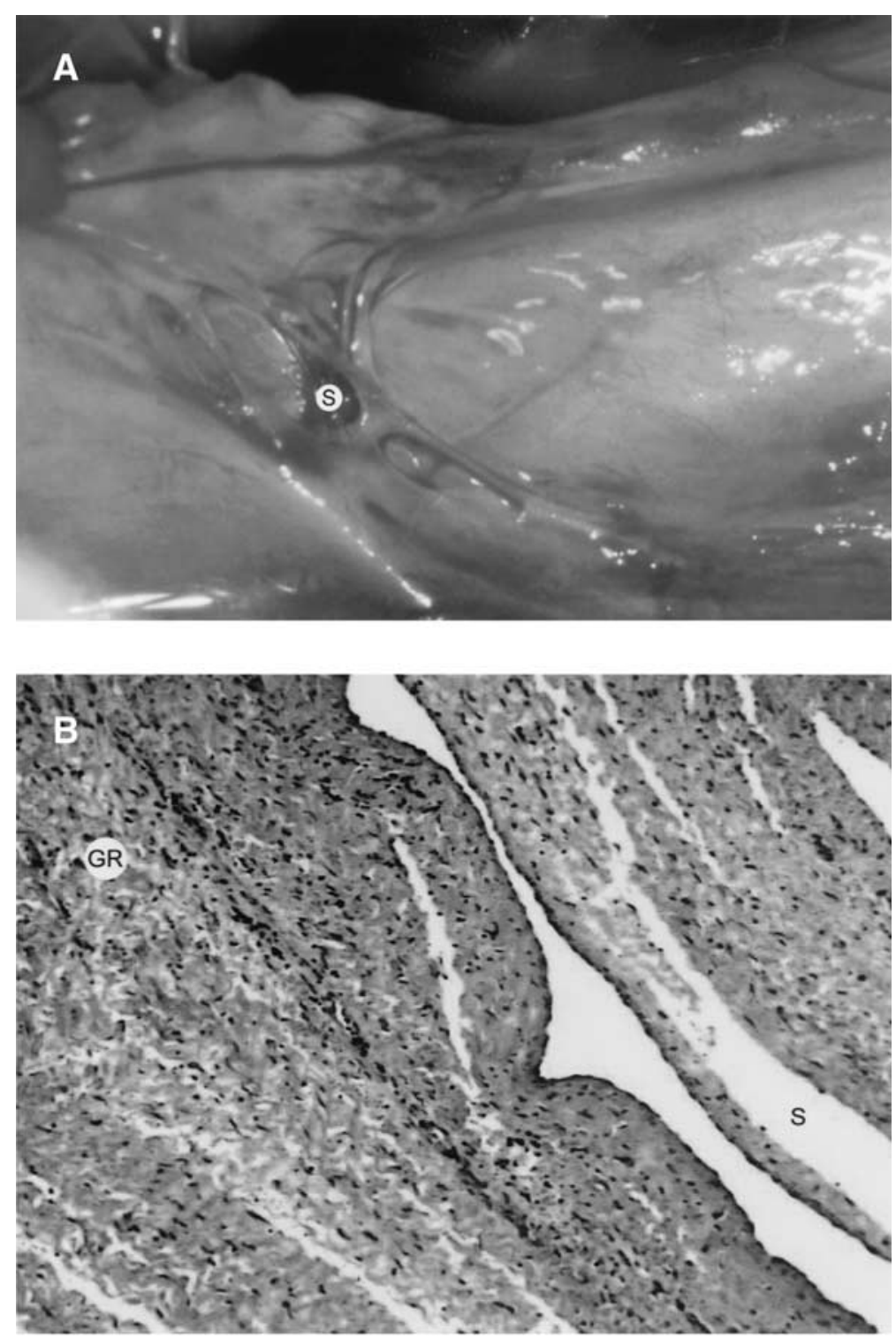

Figure 2. A, Photograph showing septae $(S)$ near superior anastomosis of opened up graft and IVC. B, Photomicrograph of graft (GR) with attached septa (S) showing similar histologic appearance.

were used. The microscopic examination of the explanted neografts revealed cellular intimal thickening, presence of mononuclear inflammatory cells and giant cells near the anastomoses and the plicated areas in the fibrocollagenous subendothelial (tunica media) layer, no inflammatory response in the adventitia, and a confluent flattened monolayer of cells (factor VIII-related antigen positive) lining $100 \%$ of the luminal surface of the graft. A rich capillary network and intramural vessels were present across the whole thickness of the graft. With van Gieson-elastin stain, (Figure 7), the elastic fibers could be easily seen in the IVC, whereas none of the grafts showed any evidence of elastic fibers. Smooth muscle of intestinal origin could be identi- fied only in 1 graft near the site of anastomosis, and it was associated with calcification and granuloma formation, probably indicative of degradation and absorption of degenerating muscle.

One occluded graft had macroscopic septae inside the lumen, 2 had minor septae, and 2 had microscopic septae, whereas 2 grafts had no septae at all. Macroscopically, the septae were made up of a fine network of fibrous strands of varied length, number, and thickness, all running in different directions, and some of them had a thin translucent membrane between different strands like a partition inside the lumen of the graft (Figure 2, A). Microscopic examination (Figure 2, B) of the septae revealed fibrocollagenous 


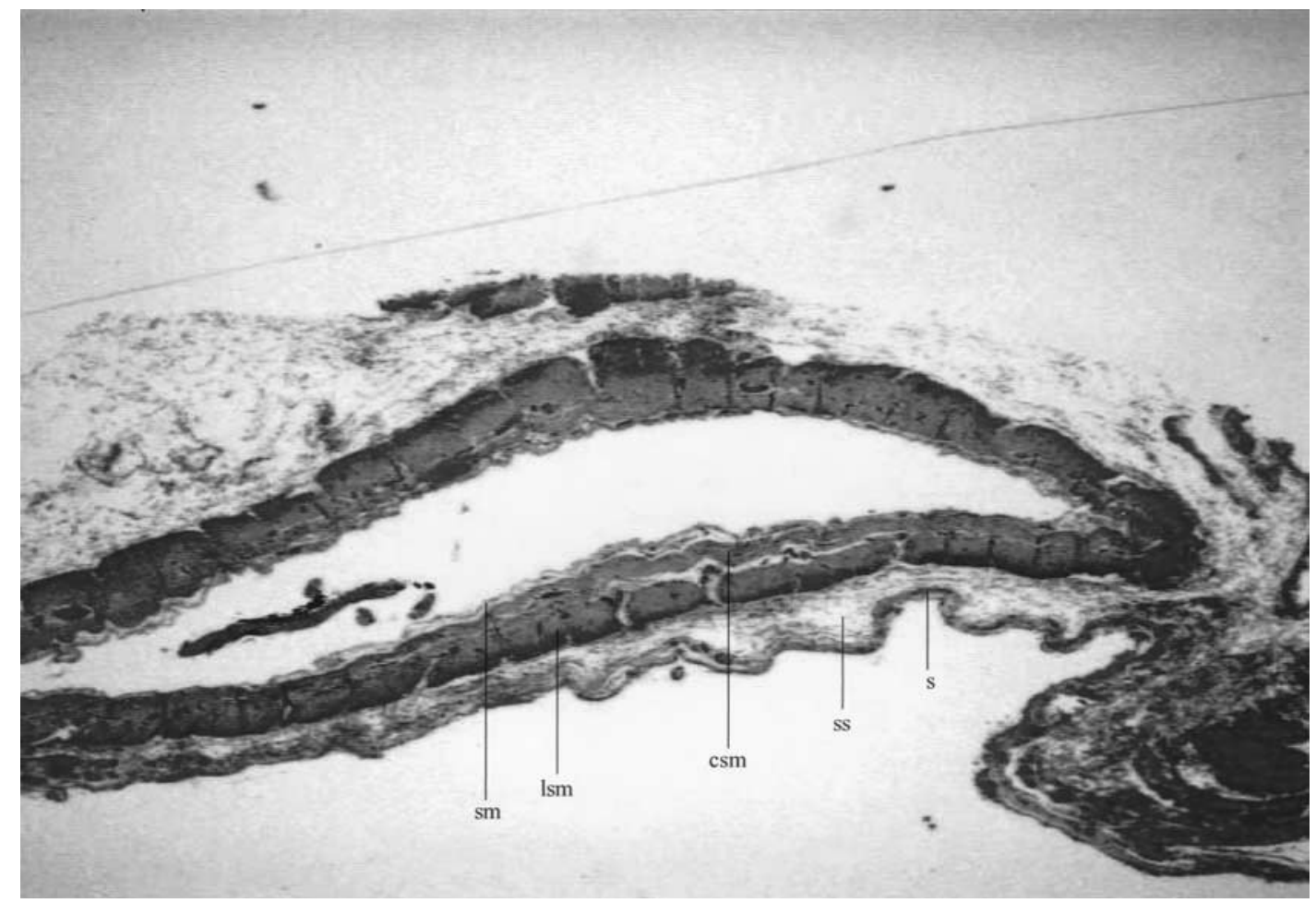

Figure 3. Photomicrograph of graft under low power before implantation showing different layers. sm, Submucosa; Ism, longitudinal smooth muscle; $c s m$, circular smooth muscle; ss, subserosa; s, serosa. (Magnification $20 \times$.)

tissue covered with a confluent monolayer of cells staining positively for factor VIII-related antigen (Figure 8). The microscopic appearances of the septae and the wall of the graft were identical except for the adventitia. In all 7 grafts, scattered proliferating fibroblasts (Figure 9) showed positive reaction to MIB1 antigen, indicative of active proliferation. Eight MIB1-positive fibroblasts per high-power field (HPF, 400×), could be seen in the animal killed at 15 days after implantation of the graft. Only 1 MIB1-positive fibroblast per HPF could be seen in the animals killed at 71, 76, 77,79 , and 148 days after implantation $(\mathrm{n}=5)$. The animal with obliterated graft, which was killed at 98 days after implantation, showed 5 MIB1-positive fibroblasts per HPF. Cells lining the luminal surface in all the grafts showed a positive reaction for factor VIII-related antigen, indicative of normal endothelial cell differentiation.

\section{Discussion}

Although the techniques of preparation, preservation, and implantation of the vascular conduits have been refined, an ideal vascular conduit has still not been found. For the pediatric population the growth potential of a vascular conduit is important, but such a conduit is not available at present. Pericardium is frequently used for repair of the pulmonary arteries and sometimes also for making a vas- cular conduit, but it is prone to calcification, ${ }^{8,9}$ shrinkage, ${ }^{8}$ and aneurysm formation. ${ }^{10}$

Matsumoto and coworkers ${ }^{1}$ in 1965 were the first to use the small intestine as a vascular conduit in experimental settings. Since then other surgeons ${ }^{2}$ have tried this also, but the results have been quite variable. Lantz and colleagues ${ }^{3-5}$ developed a technique of harvesting of submucosa of the small intestine and subsequently used it as a graft material for veins and arteries of different sizes in different sets of experiments. They reported that the submucosal graft acts like a template around which endothelium and fibrocollagenous tissue grow, and a new vascular conduit takes shape under a strict anticoagulation regimen. Miyata and coworkers $^{7}$ have described a technique of producing antithrombogenicity in the biologic materials and report successful use in vascular grafts. In short, the collagen of the biologic vascular graft is exposed to protamine sulfate, glutaraldehyde, and heparin, in that order. With this process, heparin is bound ionically to protamine, which in turn is covalently combined with the collagen. The heparin is released slowly for several weeks, rendering the vascular graft antithrombogenic. ${ }^{7}$ On the basis of work by Lantz and colleagues ${ }^{3-5}$ and Miyata and coworkers, ${ }^{7}$ Robotin-Johnson and associates $^{6}$ reported their experience with the small intestinal submucosa as a template for a growing vascular conduit in 


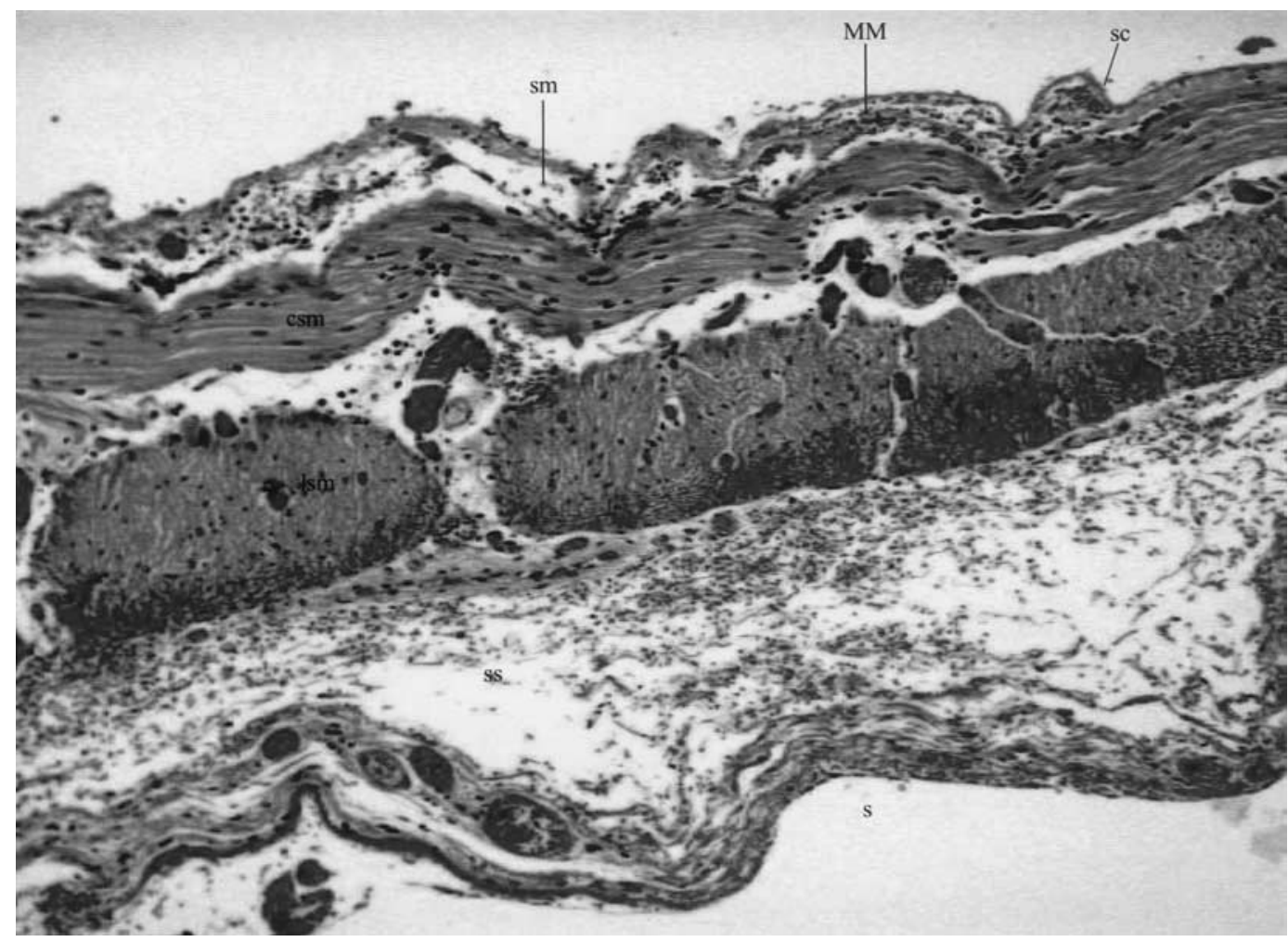

Figure 4. Photomicrograph of graft under high power before implantation showing different layers. sm, submucosa; MM, muscularis mucosae; sc, stratum compactum. (Magnification 100×.)

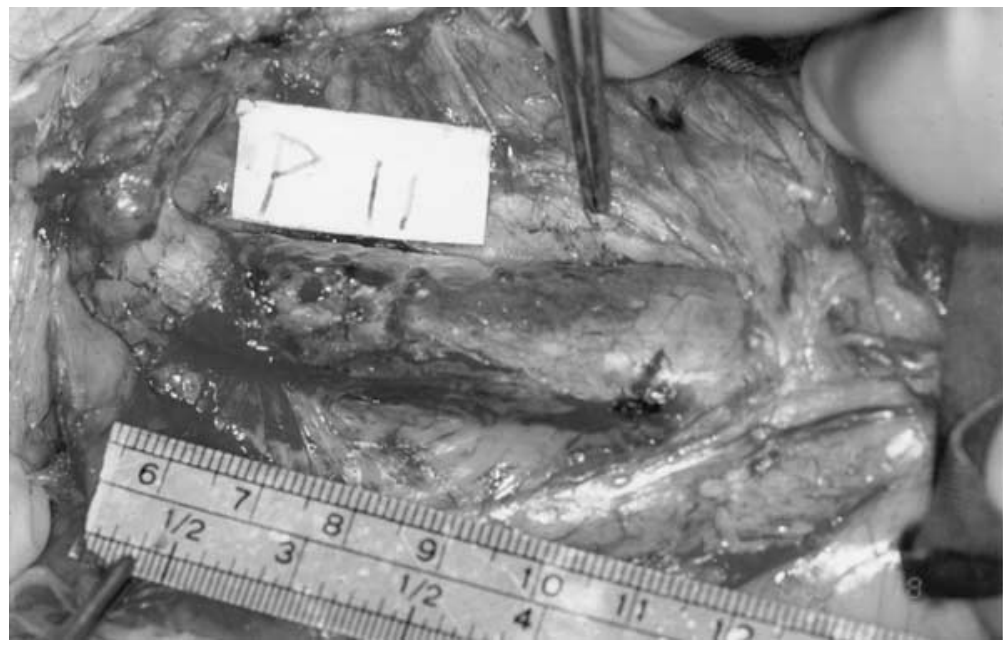

Figure 5. Nearly normal appearing SIWM graft with plicating sutures and IVC at explantation.

replacing superior vena cava with such a graft in piglets. These grafts were densely adherent to the surrounding structures, and on reviewing their results it was rather difficult to ascertain whether the grafts had actually grown, had stretched, or had passively distended, because the grafts were not plicated or trimmed at the time of implantation.
We were working on the assumption that if we could make the submucosal layer of full-thickness small intestine (without mucosa) antithrombogenic, the external layers might survive while endothelialization was taking place and we might end up with a living vascular conduit. We attempted to develop a live, growing, vascular conduit with 


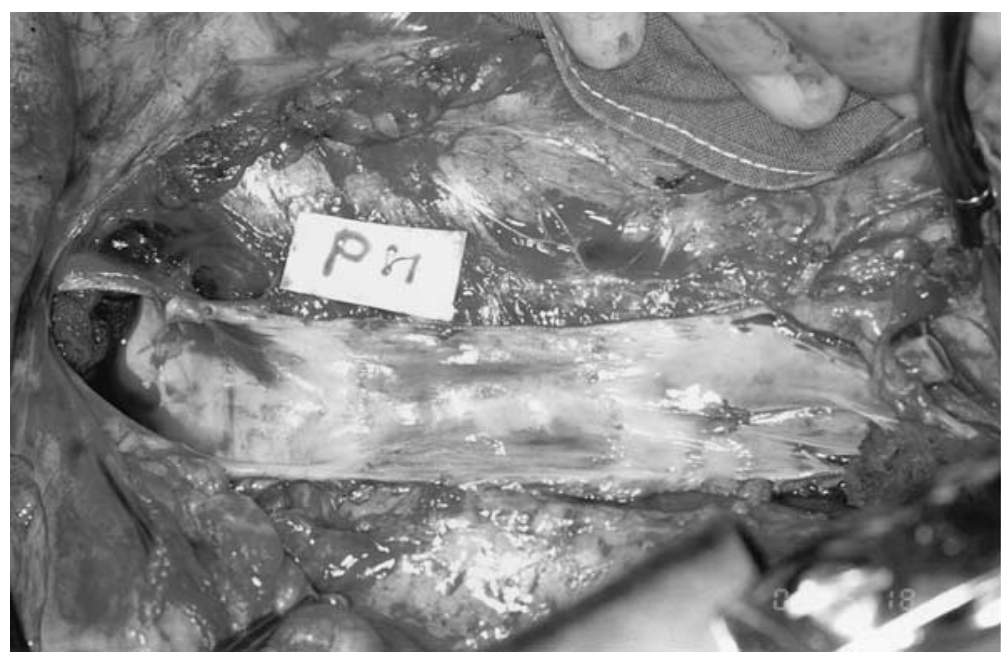

Figure 6. Opened up SIWM graft at explantation showing smooth, glistening endothelial surface and wide anastomoses.

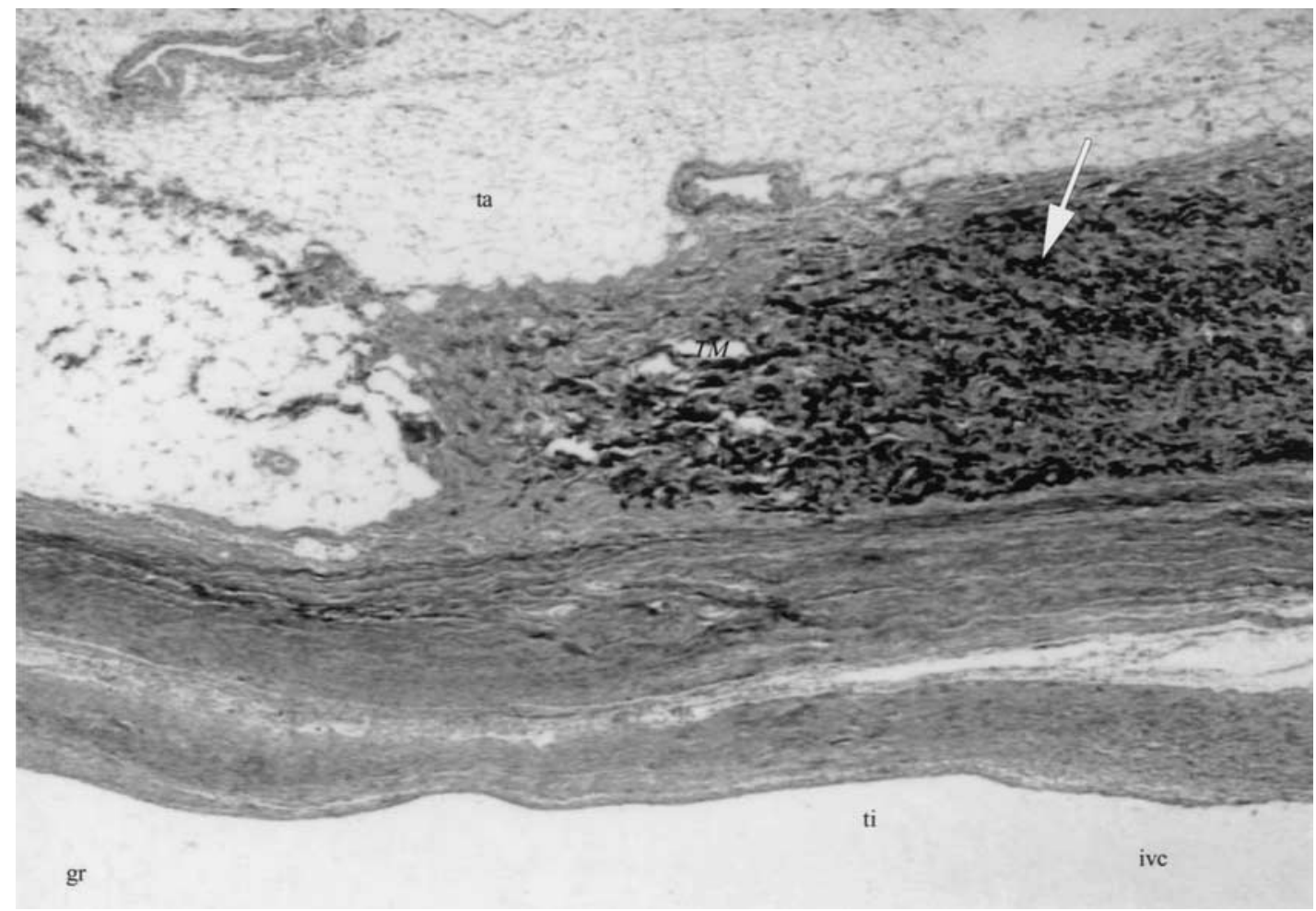

Figure 7. Photomicrograph of van Gieson-elastin stain of graft at anastomotic site showing presence of elastic fibers (black spots with arrow) in tunica media (TM) of IVC and absence of same on graft side. $t a$, Tunica adventitia; gr, graft; ti, tunica intima. (Magnification 40×.)

well-adapted tunica adventitia, tunica media with smooth muscle, and tunica intima lined with normally functioning endothelium. Antithrombogenicity was provided to the SIWM graft by modifying the technique of Miyata and coworkers. ${ }^{7}$ We used a lower concentration of glutaralde- hyde for a slightly longer duration. All reagents were applied intraluminally, with the idea of protecting the outer layers of the graft from detrimental effects of glutaraldehyde.

We wanted to ascertain three important aspects of the 


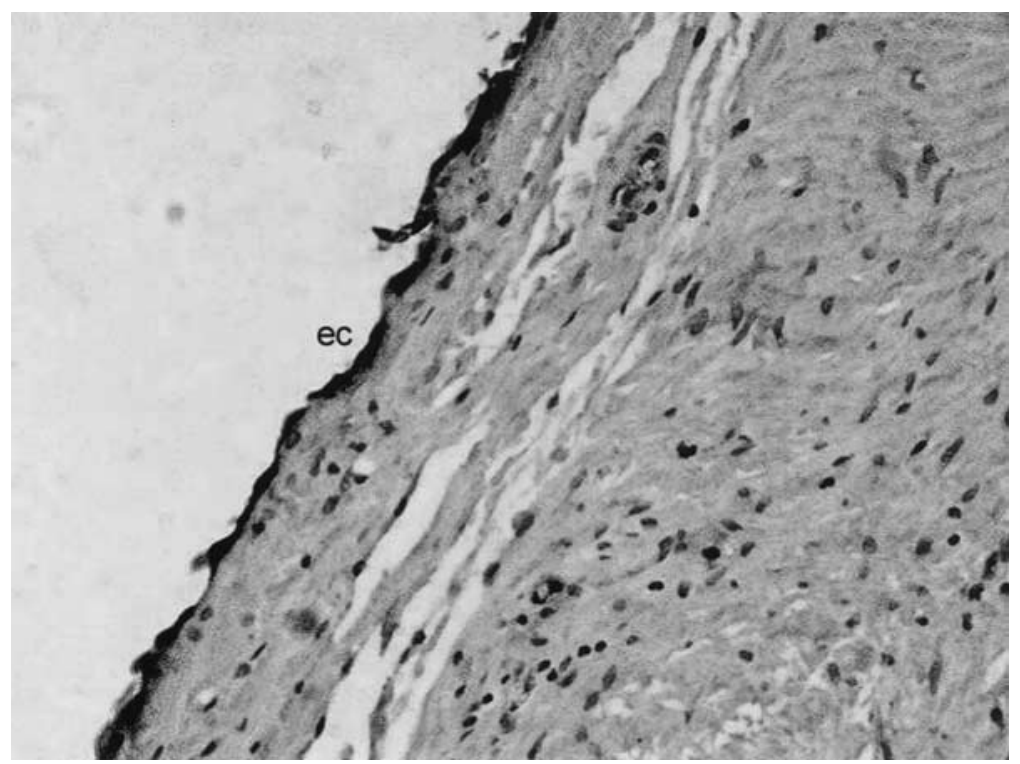

Figure 8. Photomicrograph showing endothelial cells (ec) staining positively for factor VIII-related antigen in lining of graft. (Magnification 200X).

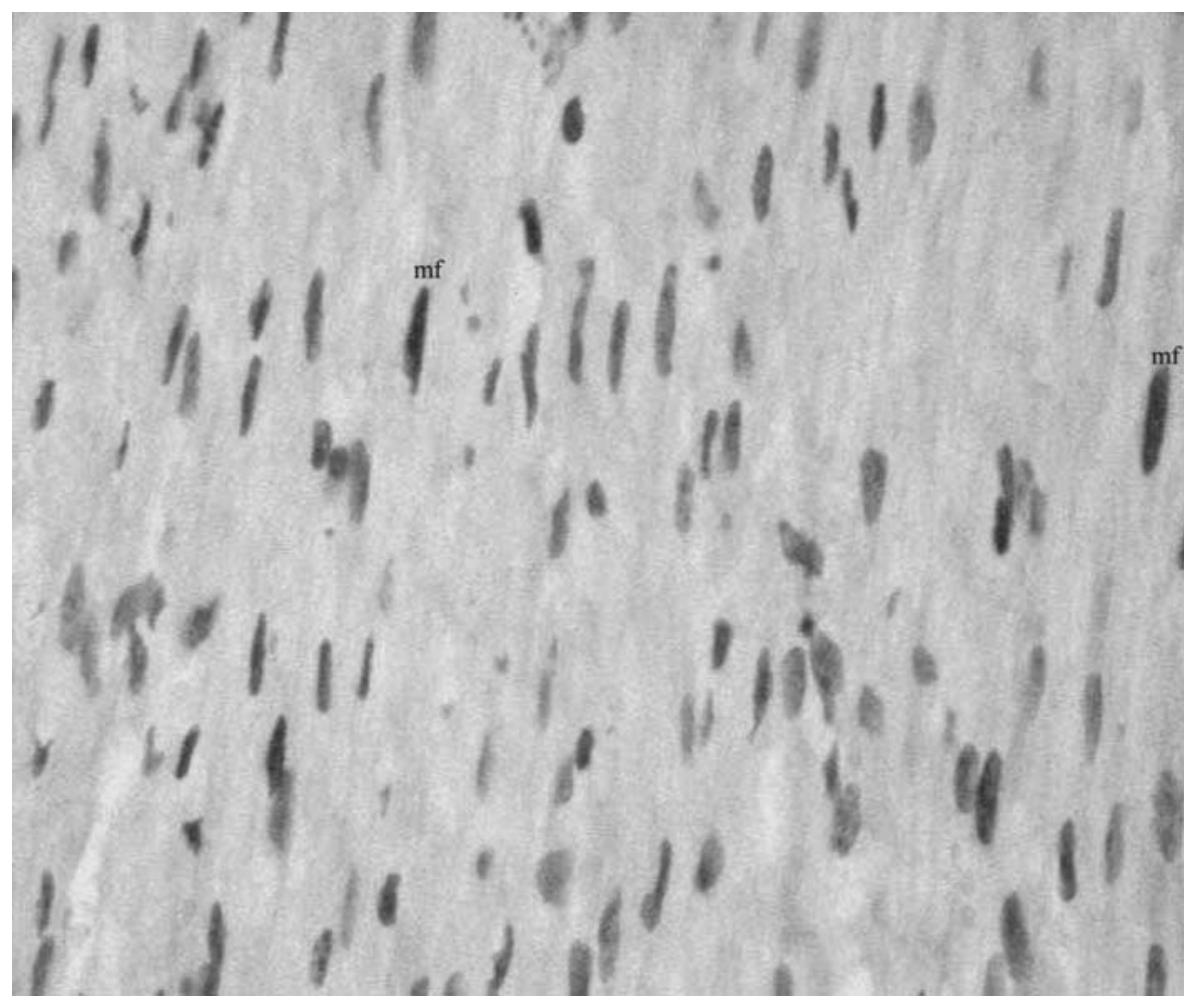

Figure 9. Photomicrograph of explanted graft showing proliferating fibroblasts (mf) staining positively for MIB1 antigen. (Magnification $400 \times$ ).

graft. First, the graft had to be vital and grow along with the animal. Second, the grafts had to be lined with normally functioning endothelial cells. Third, the neovascular wall had to be comparable to the native IVC wall and well adapted to its new environment. Regarding graft vitality, these grafts had increased in length significantly $(P<.018)$, 


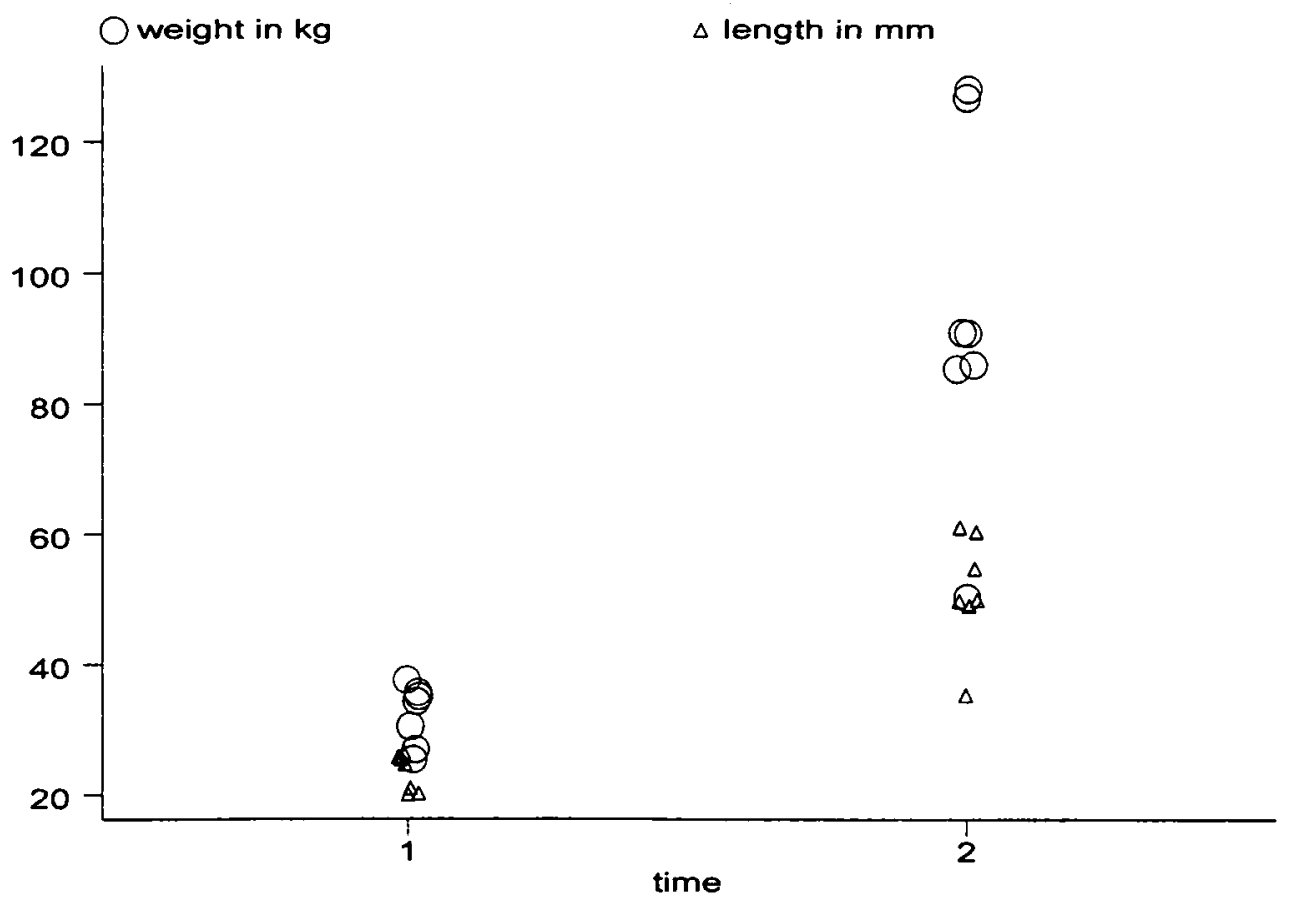

Figure 10. Graph showing weights of animals in kilograms (circles) and lengths of grafts in millimeters (triangles) at implantation (1) and explanation (2).

and in 6 animals the diameter of the graft exactly matched that of the native IVC. With MIB1 monoclonal antibody staining, proliferating fibroblasts could be demonstrated in the walls of all the grafts. Factors that point to the true growth of the graft are increases in length and diameter of the graft and increases in the size of the anastomoses with the growth of the animal. The neografts also went into spasm on handling, indicating living tissue under normal tension that was not being stretched or pulled. The growth of graft seemed to be proportional to the weight gain while the animal was growing and not while the animal was gaining weight after attaining maturity (Figure 10). One animal killed at 15 days after implantation showed a $1-\mathrm{cm}$ increase in length, and the subendothelial cell layer revealed 8 proliferating fibroblasts per HPF, indicative of active growth. On the other hand animals killed later (71, 76, 77, 79, and 148 days after implantation) had already attained maximum growth in length and diameter, and the subendothelial cell layer in these animals revealed only 1 MIB1positive fibroblast per HPF, indicative rather of stationary or dormant activity. The animal with obliterated graft, killed 98 days after implantation, had doubled the length of its graft (increase in length $2.5 \mathrm{~cm}$ ) and showed 5 MIB1positive fibroblasts per HPF, probably indicative of active cicatrization. Generalized effects of growth hormone on the growing animal and the graft and other known growth factors (such as fibroblast growth factors, vascular endothe- lial growth factor, platelet-derived growth factor, and angiogenic peptides and nonpeptides) ${ }^{11}$ may have played significant roles in the growth of the graft. The continued increasing blood flow (increased blood volume with the growth of the animal) through the IVC and the graft may have provided the mechanical stimulus for the growth of the graft. It is also postulated that the glutaraldehyde-tanned inner layers of SIWM graft, after being replaced with the fibrocollagenous tissue and inflammatory cells, got sandwiched between the outer surviving serosal layer and the inner, growing endothelial cell layer. Under the influence of previously discussed factors, the outer and the inner layers of the graft continued to grow, compelling the subendothelial fibrocollagenous layer to grow with them as a unit and also preventing cicatrization.

Regarding graft lining, all of the grafts were lined with normal appearing endothelial cells that showed a positive reaction for factor VIII antigen, indicative of normal endothelial cell differentiation. Pigs are well known to have re-endothelialization of other types of bioprosthetic and prosthetic grafts. ${ }^{12-14}$ Regarding graft structure, the IVC and the neovascular graft in 2 animals were rather similar macroscopically. Native IVC and the grafts went into spasm when manipulated, as one would expect in a normal vessel. Microscopically, what had been serosa was comparable to the adventitia connective tissue. The middle layer of the neovessel consisted of fibrocollagenous tissue in tightly and 
loosely packed layers, with some capillaries and intramural vessels. Smooth muscle was seen around intramural vessels, but the smooth muscle of the intestinal lamina muscularis externa could not be identified in 6 out of 7 grafts. It is possible that glutaraldehyde had tanned the smooth muscle layers of the small intestine during its preparation. There were mononuclear inflammatory cells and microscopic calcifications, especially near the site of anastomosis and plicated areas, probably because of foreign body (suture) granuloma. The innermost layer was composed of normal appearing endothelial cells, which showed a positive reaction to factor VIII-related antigen. The endothelial cells extended from both proximal and distal anastomotic sites to the middle of the grafts. In 1 graft major septae, in 2 grafts minor septae, and in 2 grafts microscopic septae were found, whereas there were no septae in 2 grafts. These septae may have been the result of organization of mural thrombi formed immediately after implantation of the graft. Graft function was more or less normal despite the septae. Septae may have been related to insufficient preparation in some grafts. Improved graft preparation or optimal anticoagulation could prevent this complication. There was no obvious evidence of infection of the SIWM graft in any of the animals. On the basis of the preceding discussion, we could say that the SIWM graft adapted well to the new environment.

\section{Conclusion}

Small intestine without mucosa can remodel and act like a live, growing, layered, endothelialized, nonthrombogenic (after re-endothelialization) vascular conduit in a growing pig. This material may have a potential as a growing vascular conduit in children. The septae were probably organized thrombi, the development of which could possibly be controlled by optimized anticoagulation.

We acknowledge several technicians and doctors of the cardiothoracic and pathology laboratories who assisted in our study of the vascular conduit, in particular Marco Burki, Antonio Mucciolo, Gilles Godar, and Dr Bettina Marty.

\section{References}

1. Matsumoto T, Holmes RH, Burdick CO, Heisterkamp CA, O'Connell TJ. The fate of the inverted segment of small bowel used for the replacement of major veins. Surgery. 1965;60:739-43.

2. Fraser RE, Halseth WL, Johnson B, Paton BC. Experimental replacement of the superior vena cava: autologous vein, free inverted jejunal grafts, and Dacron grafts treated with graphite-benzalkonium-heparin. Arch Surg. 1968;96:378-85.

3. Lantz GC, Badylak SF, Coffey AC, Gaddes LA, Sandusky GE. Small intestinal submucosa as a superior vena cava graft in a dog. J Surg Res. 1992;53:75-81.

4. Lantz GC, Badylak SF, Coffey AC, Geddes LA, Blevins WE. Small intestinal submucosa as a small-diameter arterial graft in the dog. J Invest Surg. 1990;3:217-27.

5. Lantz GC, Badylak SF, Hiles MC, Coffey AC, Geddes LA, Kokini K, et al. Small intestinal submucosa as a vascular graft: a review. J Invest Surg. 1993;6:297-310.
6. Robotin-Johnson MC, Swanson PE, Johnson DC, Schuessler RB, Cox JL. An experimental model of small intestinal submucosa as a growing vascular graft. J Thorac Cardiovasc Surg. 1998;116:805-11.

7. Miyata T, Noishiki Y, Matsumae M, Yamane Y. A new method to give antithrombogenicity to biological materials and its successful application to vascular grafts. Trans Am Soc Artif Intern Organs. 1983;29:363-5.

8. Mohri H, Barnes RW, Rittenhouse EA, Reichenbach DD, Dillard DH, Merendino KA, et al. Fate of autologous pericardium and Dacron fabric used as a substitute for total atrial septum in growing animals. J Thorac Cardiovasc Surg. 1970;4:501-11.

9. Gabbay S, Bartolotti U, Factor S, Shore DF, Frater R. Calcification of implanted xenograft pericardium. J Thorac Cardiovasc Surg. 1984; 87:783-7.

10. Rosenthal A, Gross RE, Pasternak A. Aneurysm of right ventricular outflow patches. J Thorac Cardiovasc Surg. 1972;63:735-40.

11. Gerritsen ME. Physiologic functions of normal endothelial cells: In: Loscalzo J, Creager MA, Dzau VJ, editors. Vascular medicine. 2nd ed. Boston: Little, Brown; 1996. p. 3-38.

12. Ratto GB, Romano P, Truiny M, Rovida S, Badini A, Zaccheo D. Glutaraldehyde-tanned mandril-grown grafts as venous substitutes. J Thorac Cardiovasc Surg. 1991;102:440-7.

13. Gouny P, Hocquet-Cheynel C, Martin-Mondiere C, Bensenane J, Bonneau M, Nussaume O. Incorporation of fibronectin-impregnated vascular prostheses in the pig, microscope study. J Cardiovasc Surg (Torino). 1995;36:573-80.

14. Parsson H, Jundzill W, Johansson K, Norgren L. Healing characteristics of polymer-coated or collagen-treated Dacron grafts: an experimental porcine study. Cardiovasc Surg. 1994;2:242-8.

\section{Discussion}

Dr Vadiyala Reddy (San Francisco, Calif). I congratulate Tucker and colleagues for their continuing efforts to develop a vascular conduit that is growing in its ideal characteristics. Since the early days of cardiothoracic surgery, the quest for ideal vascular grafts, ideal heart valves, and ideal valve conduits has been continuing. Although numerous advances have been made since Dr DeBakey's first hand-sewn homemade Dacron polyester fabric graft and Dr Haufnagel's aortic valve, the search still continues. Today there are several choices for vascular grafts as well as valves with excellent long-term outcomes. However, most still require long-term antiplatelet or anticoagulant medication. In addition, these are more suitable for adult population. For a pediatric patient, none of the existing vascular grafts or valves has the potential to grow. This by necessity then requires reoperation as a child outgrows the valve or the graft. Surgeons have tried to minimize the number of reoperations by various methods, for example by using larger grafts to account for the child's growth or by using in situ pericardial tubes. These strategies have resulted in encouraging results at midterm follow-up. The need for anticoagulant medication continues, however, and the potential for thrombosis remains. This has not been completely eliminated.

Our own experience with the use of larger polytetrafluoroethylene conduits, as well as Dr Steve Gundry's experience with in situ pericardial tubes at midterm follow-up, is encouraging. However, we still have to continue to anticoagulate these patients, and the pericardium may be prone to shrinkage and calcification in the long run.

An ideal vascular graft or valve should be biocompatible, should resist degeneration and infection, should have good handling characteristics, should be readily available in all sizes, should grow with the patient, and should not require any long-term medication, as outlined by Tucker and colleagues. Several investigators have worked on this, and small intestine as a vascular 
conduit was first used by Matsumoto in 1965. Since then work has been done by several investigators, especially Robotin-Johnson and associates of Dr Cox's group. Currently several investigators are exploring the newer techniques of endothelial cell seeding of the grafts, decellularizing the autograft or xenograft tissue so that it will form a scaffold for host tissue to invade, using biodegradable polymer scaffolds with biometric flow culture systems, or, more recently, maybe growing an entire blood vessel with genetic engineering.

Tucker and colleagues have used the small intestine with modification, as opposed to the previous investigators. They have left the entire small intestine and taken out only the mucosa, as opposed to using only the intestinal submucosa itself. There was a modification of Miyata and coworkers's technique in using antithrombogenicity. With these modifications they operated on 8 piglets, 7 of which survived. It is notable that 1 out of the 7 grafts became blocked, which is about $15 \%$ statistically. Another concern is that 2 of 6 grafts had macroscopic septae and another 2 had microscopic septae. Tucker and colleagues actually attributed 1 of the blocked grafts to this possible formation of septae. With the short-term follow-up of 3 months, these results do not appear to me to be encouraging. Although Tucker and colleagues demonstrated that the conduits had grown, the graft appeared to fall short of the ideal requirements. At pathologic examination the authors showed fewer adhesions than with the subintestinal mucosa. These grafts were lined by endothelial cells, as was demonstrated by previous authors, and growth seems to have been documented.

I congratulate your group on your continuing efforts in this regard, Dr Tucker. However, I have four questions. First, this technique involves an abdominal operation on the patient, with the potential for infection that has been documented previously. Would you consider this to be a serious problem, especially if it requires multiple operations? Second, why did you modify the technique of antithrombogenicity as described by Miyata and coworkers without any scientific basis for doing so? Third, the septae are obviously a concern. Is it something to do with your plication technique to decrease the lumen of the graft, as opposed to doing an excision and reanastomosing this graft to appropriate size? Fourth and most importantly, your technique does not create a graft with any shelf life and requires an operation to retrieve the graft. Please share your comments on this.

Dr Tucker. Thank you for the kind comments, Dr Reddy. Regarding your first question about infection, I know that this is a contaminated graft, a clean-contaminated graft. The jejunum is harvested very near the duodenal-jejunal junction, and it is washed thoroughly initially and while being prepared with different solutions. It is also treated by glutaraldehyde. As you know, sometimes the valves have been prepared with glutaraldehyde, and glutaraldehyde acts as a sterilizing solution also. In this study, although clinically or macroscopically there was no evidence of infection, I cannot say whether grafts were infected because no tests were done. If the graft is harvested from the extreme proximal jejunum, I do not consider it to be a serious problem.

In response to your second question, we modified the technique of Miyata and colleagues because we wanted to get a live and growing graft. One has to implant live tissue to get a live graft. We a used lower concentration of glutaraldehyde intraluminally with the idea of protecting the outer layers of the intestine from the detrimental effects of glutaraldehyde. In all the grafts fibrous tissue replaced the inner layers of intestine. The graft was lined by endothelium on the inside and living serosal tissue on the outside, allowing the graft to grow and preventing formation of stricture or stenosis.

Regarding septae, I think that the septae have occurred because of either inadequate antithrombogenesis or inadequate anticoagulation after operation. In the future, we would refine our techniques for the preparation, imparting antithrombogenesis to the graft and maintaining an anticoagulation regimen after operation.

Regarding the lumen of the graft, what was your comment?

Dr Reddy. There is no shelf life to your graft. You have to retrieve it at the time of the operation.

Dr Tucker. Yes. If you want to keep it alive, you have to retrieve and implant it at the same time. If you leave it for a longer time, it may not remain viable.

Dr Reddy. It is a disadvantage in terms of availability of the grafts. One of the important things I noticed was that at 1 month the graft that was blocked was completely patent and there were no septae according to IVUS.

Dr Tucker. I would not say that it was completely patent. During IVUS, there was a lot of reaction seen around the graft. The lumen appeared smaller, so we could predict that something wrong was going on.

Dr John Benfield (Los Angeles, Calif). The intestine needs a blood supply to survive. You have shown slides that indicate that the muscularis of the intestine survived without an apparent blood supply. How did that happen?

Dr Tucker. The grafts did not show any intestinal smooth muscles. The intestinal smooth muscles were replaced with fibrous tissue. There were only endothelial cells, fibrous tissue, and serosa. The layers that were fixed or treated with glutaraldehyde perished and were replaced with fibrous tissue. Serosa survived the ill effects of glutaraldehyde and received its nutrition from oxygen from the blood it carried. 